\title{
The U5.0 Undulator Design for the Advanced Light Source at LBL
}

E. Hoyer, J. Chin, K. Halbach, W. Hassenzahl, D. Humphries, B. Kincaid, H. Lancaster, D. Plate, and R. Savoy

\author{
Accelerator and Fusion Research Division \\ Lawrence Berkeley Laboratory \\ 1 Cyclotron Road \\ Berkeley, California 94720
}

August 1989 


\title{
THE U5.0 UNDULATOR DESIGN FOR THE ADVANCED LIGHT SOURCE AT LBL*
}

\author{
E. Hoyer, J. Chin, K. Halbach, W. Hassenzahl, D. Humphries, B. Kincaid, H. Lancaster, D. Plate, \\ R. Savoy
}

\author{
Accelerator and Fusion Research Division \\ Lawrence Berkeley Laboratory \\ 1 Cyclotron Road \\ Berkeley, California 94720
}

The U5.0 undulator, currently under design, is the first in a series of insertion devices planned for the Advanced Light Source at LBL. U5.0 parameters include a $5 \mathrm{~cm}$ period, $5 \mathrm{~m}$ length with a $0.837 \mathrm{~T}$ maximum field at a $14 \mathrm{~mm}$ gap. A hybrid configuration utilizing Nd-Fe-B permanent magnet material and Vanadium Permendur poles is used for the magnetic structure. Construction is modular with many pole assemblies attached to a pole mount, which in turn is fastened onto one of the backing beams. Vertical field integral correction at the ends is with permanent magnet rotators. The support structure features a 4-post configuration, a rigid base with 3 kinematic floor supports and 2 rigid $5 \mathrm{~m}$ long backing beams that fit within the $2.4 \mathrm{~m}$ high accelerator enclosure. The drive system is computer controlled utilizing a stepper motor and shaft encoder coupled to a roller-screw/nut and chain drive train. Vacuum chamber design is a rigid configuration with a $10 \mathrm{~mm}$ vertical by $218 \mathrm{~mm}$ horizontal aperture of $5.5 \mathrm{~m}$ length. Chamber fabrication features a two-piece welded chamber of 5083 H321 aluminum. Pumping is with ion and titanium sublimation pumps.

\footnotetext{
"This work supported by the Director, Office of Energy Research, Office of Basic Energy Sciences, Materials Sciences Division, of the U. S. Department of Energy under Contract No. DE-AC03$765 F 00098$.
} 


\section{INTRODUCTION}

The U5.0 Undulator is the first of a family of insertion devices to be built and installed on the Advanced Light Source (ALS) at Lawrence Berkeley Laboratory. This 98 period, $5 \mathrm{~cm}$ period, 5 meter long device is designed to produce synchrotron radiation in an energy range from $52 \mathrm{eV}$ to $1,500 \mathrm{eV}$ [1]. Operating over this range requires $0.837 \mathrm{~T}$ Beff at minimum gap to reash the lowest energy and the ability to produce a high brightness in the 1st, 3rd, and 5th harmonics. The former can be accomplished by designing a robust magnetic structure, while achieving the latter depends on characteristics of the accelerator and establishing appropriate error tolerances for the U5.0 design.

Some parameters and tolerances for U5.0 are given in Table I [2]. These characteristics were established to fully utilize the potential of the ALS. Tolerances in the table were set by calculations of effects on spectral performance [3].

The U5.0 Undulator is shown in Figure 1. The major subsystems identified are: the magnetic structure, which includes the hybrid pole assemblies mounted on pole mounts that are attached to the 5 meter long backing beams; the support and drive system, which includes the framework for supporting the magnetic structure and the mechanism for opening and closing the magnetic gap; and the vacuum system, which includes a 5.5 meter long vacuum chamber and its associated pumping system.

Present plans call for the initial compliment of insertion devices to include three 5 meter long undulators, U3.9, U5.0, and U8.0, and one 2.5 meter long wiggler, W13.6. The approach taken for the design of the initial compliment of ALS insertion devices has been toward a generic insertion device design with the objective of reducing engineering and fabrication costs and for easier maintainability. This leads to similar undulator and wiggler components that include the backing beams, support structures, drive systems, control systems, vacuum chambers, and pumping systems.

The design, fabrication, testing, and installation of the U5.0 Undulator takes advantage of our experience with the BL VI and BL X Wigglers that are now operational at SSRL [4], [5].

\section{MAGNEMC STRUCTURE}

The magnetic structure includes the periodic magnetic structure, the end rotators, the $5 \mathrm{~m}$ long backing beams and auxiliary tuning coils.

The U5.0 Undulator incorporates a hybrid magnetic configuration consisting of Nd-Fe-B magnetic blocks and vanadium permendur poles. The hybrid is chosen because of several advantages over the pure current sheet equivalent material (CSEM) design.

- The field is dominated by the characteristics of the poles, which can be made very uniform both in size and magnetic performance. 
- The errors in magnetic moments of the blocks can be averaged by sorting the blocks for the poles.

- Errors in the total magnetic moment of all the blocks on a pole have little effect on the electron beam, or the spectrum, because they contribute equally to adjacent poles and produce no electron beam steering.

- The peak field at each pole can be tuned by a small amount.

- A higher peak field is achievable.

The periodic magnetic structure consists of Neodymium-Iron-Boron blocks and vanadium permendur poles. The configuration is conventional with a rectangular pole and Nd-Fe-B block array. $A$ one half period section of the structure is shown in Figure 2. This configuration is chosen for its simplicity, ease of manufacture and high performance. The principal objective of the design effort is to develop a magnetically well behaved structure which yields a high value of Beff for mid-plane fields. The design approach utilizes Halbach 3-D Hybrid Theory and 2-D modeling with PANDIRA [6], [7].

Mid-plane field performance optimization is accomplished by fixing the operating point of the Nd-Fe-B and determining the pole thickness $\left(T_{\text {pole }}\right)$, which maximizes the value of $B_{\text {eff: }}$

$$
B_{\text {eff }}^{2}=B_{1}^{2}+\frac{1}{3^{2}} B_{3}^{2}+\frac{1}{5^{2}} B_{s}^{2}+\cdots
$$

where $B_{1}$ is the amplitude of the fundamental and $B_{3}$ is the amplitude of the 3rd field component, etc.

To verify the actual periodic magnetic structure design performance, which is based on magnetic design calculations and the Nd-Fe-B material selected, a test pole assembly will be fabricated and tested.

To avoid steering perturbations of the beam as it travels through the insertion device, it is necessary to control the configuration of the fields at the ends. Because of concerns about dimensional instability caused by coil heating effects, no end coils are used in this design. Instead, the basic end pole design utilizes a system of Nd-Fe-B rotors to fine tune the fields at the ends of the insertion device. These rotors allow precise and convenient adjustment of end fields and their position can be set manually or be remotely controlled.

As shown in Figure 3, there are four rotors at each end of the device, two above the horizontal midplane and two below. There is an additional fixed quantity of Nd-Fe-B at the location of each rotor. Beyond the second rotor from the end, the structure is periodic with the full quantity of Nd-Fe-B between each pair of poles. Gap dependent errors at the ends are small, thus the objective is to 
determine a fixed orientation for all rotors that minimizes the steering errors introduced by the end correctors over the entire range of gaps. Rotor adjustment is accomplished by manually varying the orientation of the rotors, then locking them in place at their optimum settings.

Figures 2 and 4 show the proposed U5.0 magnetic structure which includes:

- Half period pole assemblies consisting of an aluminum keeper, a vanadium permendur pole (8 $\mathrm{cm}$ wide $\times 6 \mathrm{~cm}$ high $\times 0.80 \mathrm{~cm}$ thick), Nd-Fe-B blocks $(3.5 \mathrm{~cm} \times 3.5 \mathrm{~cm} \times 1.70 \mathrm{~cm}$ in the orientation direction). Pole assembly fabrication is planned to be similar to that used on the BL VI and BL $X$ wiggler poles.

- Assembly sections each includes approximately 32 pole assemblies, and a pole mount, fabricated from $\mathrm{jig}$ plate aluminum-for mounting and accurately positioning the half period pole assemblies. Each assembly section is kinematically mounted onto a backing beam.

- Stress relieved steel backing beams hold the magnetic structure components and provide magnetic shielding. These $5 \mathrm{~m}$ long beams are rigid, with a deep, $81 \mathrm{~cm}$, web and a $89 \mathrm{~cm}$ wide flange to minimize systematic gap variations, which cause a loss in spectral performance.

- Dipole and steering will be designed and implemented if required.

\section{SUPPORTIDRIVE SYSTEM}

The support/drive system includes the support structure that provides the framework for holding the magnetic structure and drive train and the drive system which opens and closes the magnetic gap.

The support structure, shown in Figure 1, is designed to support a maximum magnetic load of 84,000 Ibs, the loading due to a $1.85 \mathrm{~T}$-field, $-10 \mathrm{~cm}$ pole width undulator. The support structure design is compatible with the storage ring tunnel and the adjacent beamlines, can accommodate the vacuum system, and has an alignment method, and has a floor support system.

The 4-Post support structure was selected over the C-Frame type configuration for the following reasons:

- Greater tunnel walkway clearance.

- Gap deflection less due to more rigid support structure.

- No pole rotation due to symmetrical loading.

- Better access for assembly of components.

The main advantages of a C-Frame-type structure are that it would be open on its outer radius, which would allow magnetic measurements with an external measurement system, and allows insertion device installation with the vacuum chamber in place. 
The support structure consists of a base onto which four vertical posts are mounted. Four horizontal beams, which carry the backing beams of the magnetic structure, are mounted on these posts. These four horizontal beams pass through the webbing of the backing beams to limit the overall height of the support structure to less than 8 feet.

The base is a welded assembly contzining three $y$-axis leveling mounts for a kinematic support system; $x \& z$ axis adjustments are also provided at each leveling mount. This arrangement provides a satisfactory range of adjustments for all six degrees of freedom as needed for installation and alignment. The four vertical posts and four horizontal beams will be bolted to the support frame. This modular arrangement allows for ease of fabrication, installation, calibration and servicing. All subassemblies are to be stress-relieved before final machining to minimize material creep.

A magnetic load compensating spring system is provided to buck the gap dependent magnetic load [8]. The spring assembly consists of several stacks of belleville washers and one helical compression spring selected to match the magnetic load to within $20 \%$. This compensating spring system provides the following advantages:

- Reduced system friction which gives better positional response from the drive system.

- Msinimum motor load holding torque at any magnet gap required, which gives stationary stability when the null position is reached. Motor current can be turned off or reduced to minimize motor heat-up.

- Elimination of "lifting" when the magnetic load exceeds the gravitational beam weight of the lower backing beam.

- Reduced structure load which gives better gap reproducibility.

The drive system design capabilities include opening the magnetic gap with an 84,000 lbs magnetic load; a step resolution $<1.02 \mu \mathrm{m}$ (based on $1 / 10$ of the 5 th harmonic for a U3.65 undulator); a velocity $>2.31 \mathrm{~mm} / \mathrm{sec}$ (based on a scan rate of 1 bandwidth/sec for an $11 \mathrm{~cm}$ period device) and a magtıëic gap range of $1.4 \mathrm{~cm}$ to $21.6 \mathrm{~cm}$.

The backing beams are supported by Transrol roller screws of $2 \mathrm{~mm}$ pitch. These roller screws are all connected together with a system of roller chains, sprocket wheels and spring-loaded idler sprocket wheels. The gearbox, a 30:1 reduction unit, and a stepper motor drives the system. An absolute rotary encoder is coupled to a Transrol roller screw shaft to read the absolute position of the magnet gap.

\section{CONIROL SYSTEM}

The goal of the insertion device control system (IDCS) is to provide sufficient position accuracy, resolution, velocity, and range for the motors and encoders to accommodate the full compliment of 
anticipated insertion devices. If possible, this should be accomplished using the same hardware and software so as to minimize the development effort and maintenance costs. The motor controller should be commercially available or assembled with commercially available modules from a well based manufacturer. In addition the IDCS must provide for control and monitoring of dipole and steering correction coil power supplies, as well as for gap dependent rotator positioning, if needed.

The IDCS is to be integrated into the overall accelerator computer control system. The insertion device gap must be capable of being controlled (via request to the accelerator control system) and monitored by the experimenter using the generated synchrotron radiation. During development the insertion device is to be capable of being manipulated through the IDCS by a local computer so that the necessary control and monitor algorithms can be determined.

The commercial motor controller, which is interfaced through an IEEE-488 buss, can control the gap indexer and, if needed, all four rotator indexers. Provisions for interfacing limit switches are included in the indexer as well as the ability to compensate for backlash and to program acceleration and deceleration curves. The indexers can be programmed to microstep the motors with as many as 25,000 steps per revolution. With the designed gear ratios 2,000 steps per revolution corresponds to about 0.1 $\mu \mathrm{m}$ of gap motion per step. An absolute position encoder is used to give accurate return-to-position information without the return-to-home required when using an incremental position encoder. The gap encoder is of the rotary type and is connected to the gap lead screw as close as possible to the gap. It covers the total range of $20.2 \mathrm{~cm}$ with a resolution of $0.1 \mu \mathrm{m}$.

\section{VACUUM SYSTEM}

A plan view of the U5.0 undulator vacuum system is shown in Figure 5. Two vacuum chambers are required for ALS operation, one chamber for commissioning and one for dedicated operation. The commissioning chamber is to have a $2.0 \mathrm{~cm}$ vertical aperture and a cross section that closely follows the cross section of the storage ring sector chamber. The dedicated operation chamber is to be of rectangular aperture with a $1.0 \mathrm{~cm}$ vertical gap.

Both chambers are to be fabricated from two aluminum plates, alloy $5083 \mathrm{H} 321$. This material is chosen over stainless steel for economy and that there is no difference in the radiation dosage to the CSEM material. This alloy is also the same material chosen for the storage ring sector chambers so fabrication should benefit from fabrication experiences currently under way on the sector chambers. Both chambers are machined to provide a minimum horizontal gap of $21.8 \mathrm{~cm}, 32 \mathrm{~cm}$ to the inside circumference of the storage ring for the circulating beam aperture and $18.6 \mathrm{~cm}$ to the outside circumference to prevent bending magnet radiation from hitting the wall of the vacuum chamber. Both 
chambers also have an ante chamber along the complete length and to the outside circumference for increased pumping conductance. Most of the unwanted synchrotron radiation is absorbed by the photon stop located at the exit end of the chamber. The outside of both chambers have pockets machined into them for the magnet poles to achieve a minimum magnetic gap of $2.4 \mathrm{~cm}$ for commissioning and $1.4 \mathrm{~cm}$ for dedicated operation. The two ilalves are welded together in a manner to facilitate the assembly and welding of all the required ports and to minimize warping.

The vacuum pumping system proposed consists of six combirztion $600 \mathrm{l} / \mathrm{s}$ titanium sublimation and $50 \mathrm{l} / \mathrm{s}$ ion pumps of commercial manufacture, which give a net pumping speed of $173 \mathrm{l} / \mathrm{s}$ each at the antechamber, and an ALS Absorber pump of $1450 \mathrm{l} / \mathrm{s}$ capacity for a total antechamber pumping speed of 2,500 I/s. The beam aperture pressure distribution for the vacuum chamber was estimated using a thermal outgassing rate of $10^{-11}$ Torr $1 / \mathrm{s} \mathrm{cm}^{2}$ and a molecular production rate, due to photon induced desorbtion, of $10^{-5}$ molecules/photon with photons of energies greater than $10 \mathrm{eV}$ considered [9]. The pressure distribution obtained, based on $1.9 \mathrm{GeV}-400 \mathrm{~mA}$ storage ring operation, is $3 \times 10^{-10}$ Torr in the insertion device vacuum chamber beam aperture [10].

\section{REFERENCES}

[1] An ALS Handbook, Rev. 2, PUB-643, April 1989.

[2] U5.0 Undulator Conceptual Desien Report, Draft, June 1989.

[3] R. Savoy et al, Calculatica of Magnetic Error Fields in Hybrid Insertion Devices SRI 89, to be published in August 1989.

[4] E. Hoyer, et al., The Beam Line VI REC-Hybrid Wiggler for SSRL, IEEE Trans. NS-30, (1983), p 3118.

[5] E. Hoyer, et al., The Beam Line X Nd-Fe-Steel Hybrid Wiggler for SSRL, IEEE Catalogue No. 87, Chap. 2387-9, (1987), p 1508.

[6] K. Halbach, Insertion Device Design. LBL Lecture Series, October 1988-March 1989, (unpublished).

[7] K. Halbach, et al., developed PANDIRA, an improved version of POISSON, which allows solution of permanent magnet and residual field problems; POISSON is an improved version of TRIM (A. M. Winslow, J. Computer Phys. 1, 149, 1967).

[8] J. Chin, Magnetic Load Compensating Springs, LBL Engineering Note M6829, (April 1989).

[9] E. Hoyer, Pumping System Desien Calculations, LBL Engineering Note M6821, (March 1989).

[10] E. Hoyer, CDR Vacuum Chamber Pressure Distribution, LBL Engineering Note M6844, (May 1989 ). 


\section{FGURES}

Figure 1: U5.0 Undulator Design

Figure 2: $\quad$ U5.0 Half Period Pole Assembly

Figure 3: End Rotator Configuration

Figure 4: U5.0 Magretic Structure Configuration

Figure 5: Vacuum System Layout 
Table I. U5.0 Parameters.

Period

Overall Length

Number of Periods

$5.0 \mathrm{~cm}$

Number of Poles

$5.0 \mathrm{~m}$

End Sequence

Maximum Operation Field

Minimum Operational Gap

Usable Spectral Harmonics

98

197

Pole Height

Pole Widin

* of Blocks per Pole

Block Material

Gap Variation-Single Pole

$-1 / 2+1$

$0.837 \mathrm{~T}$

$1.4 \mathrm{~cm}$

1st, 3rd, \& 5th

$6 \mathrm{~cm}$

$8 \mathrm{~cm}$

6

Nd-Fe-B

22 microns

54 microns

Gap Variation-Systematic

$0.28 \mathrm{mrad}$

Single Pole Tilt

$0.80 \mathrm{~cm}$ :

Pole Thickness Variation

50 microns

Block Easy Axis Orientation-Center Block

$+/-1.3$ degrees

Block Easy Axis Orientation-Side Blocks

$+/-2.3$ degrees 


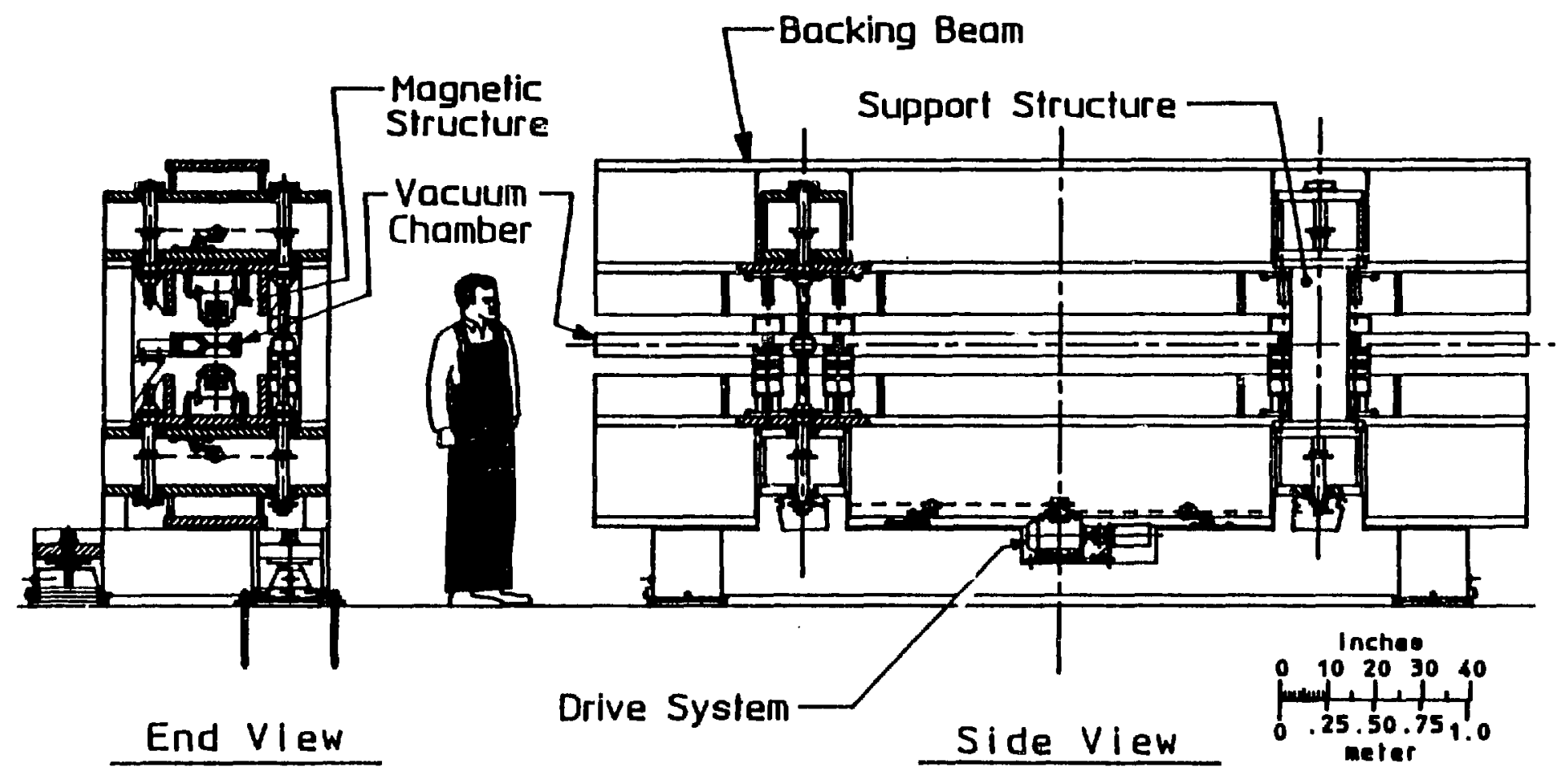

Figure 1 


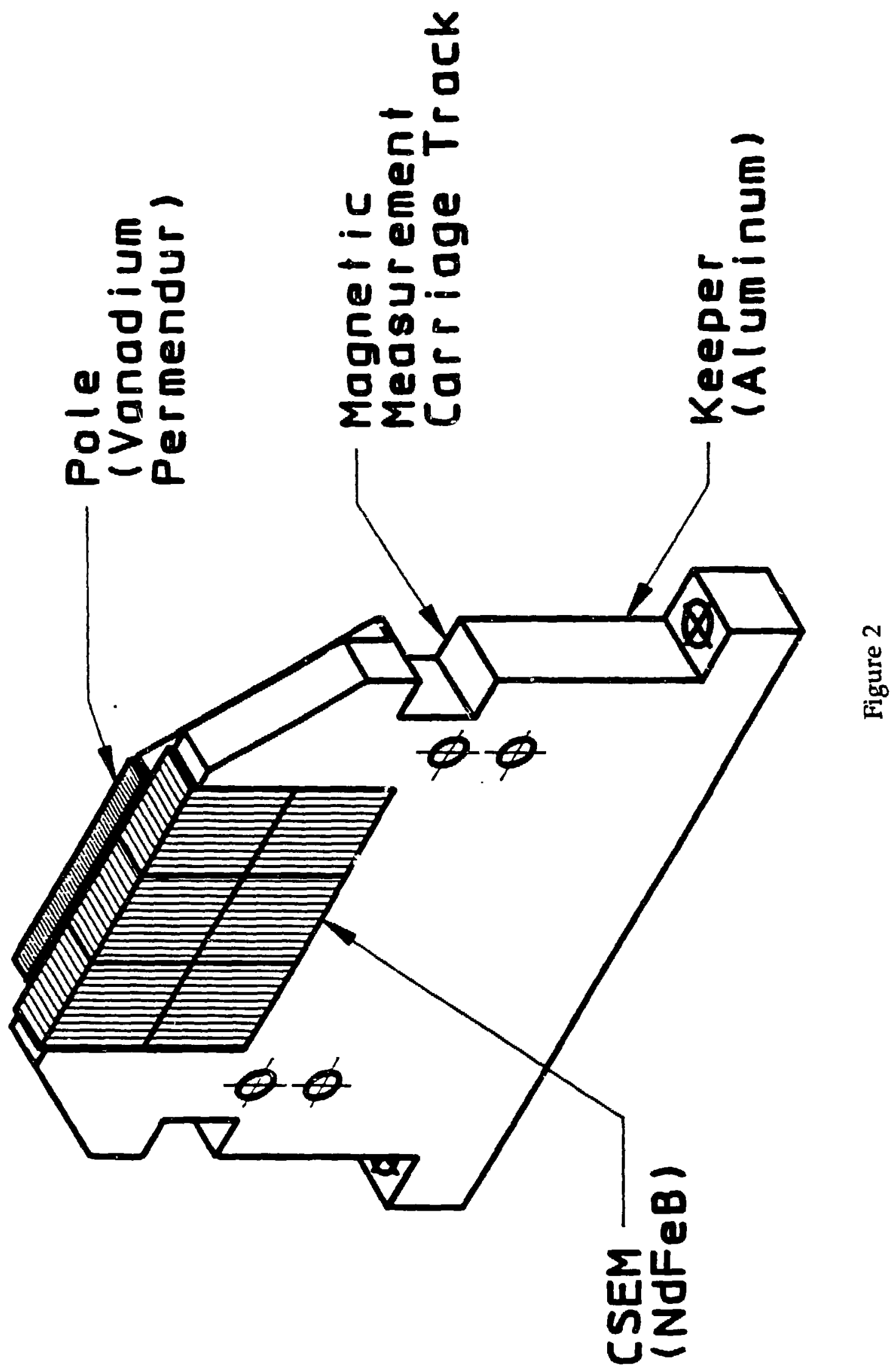




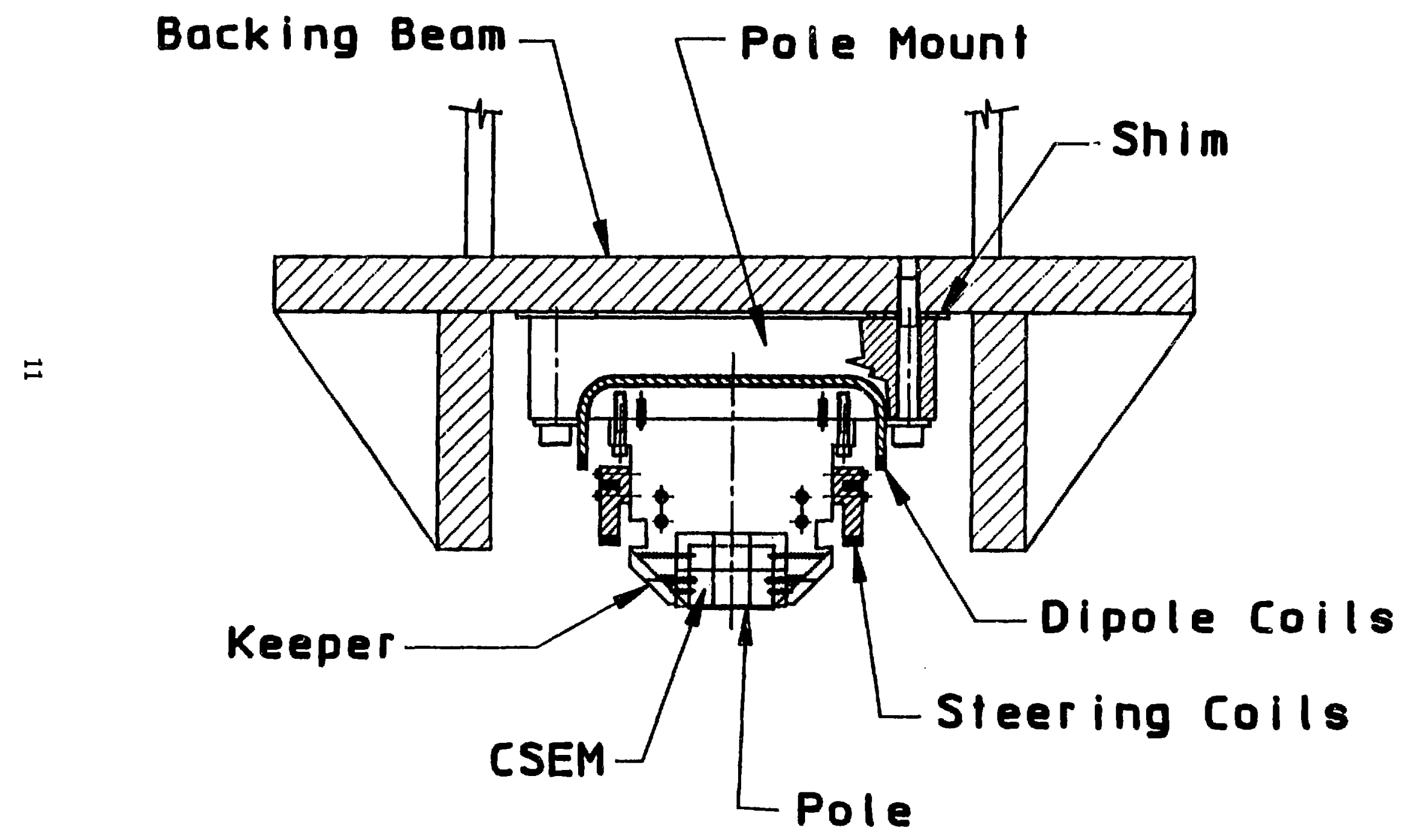

Figure 3 

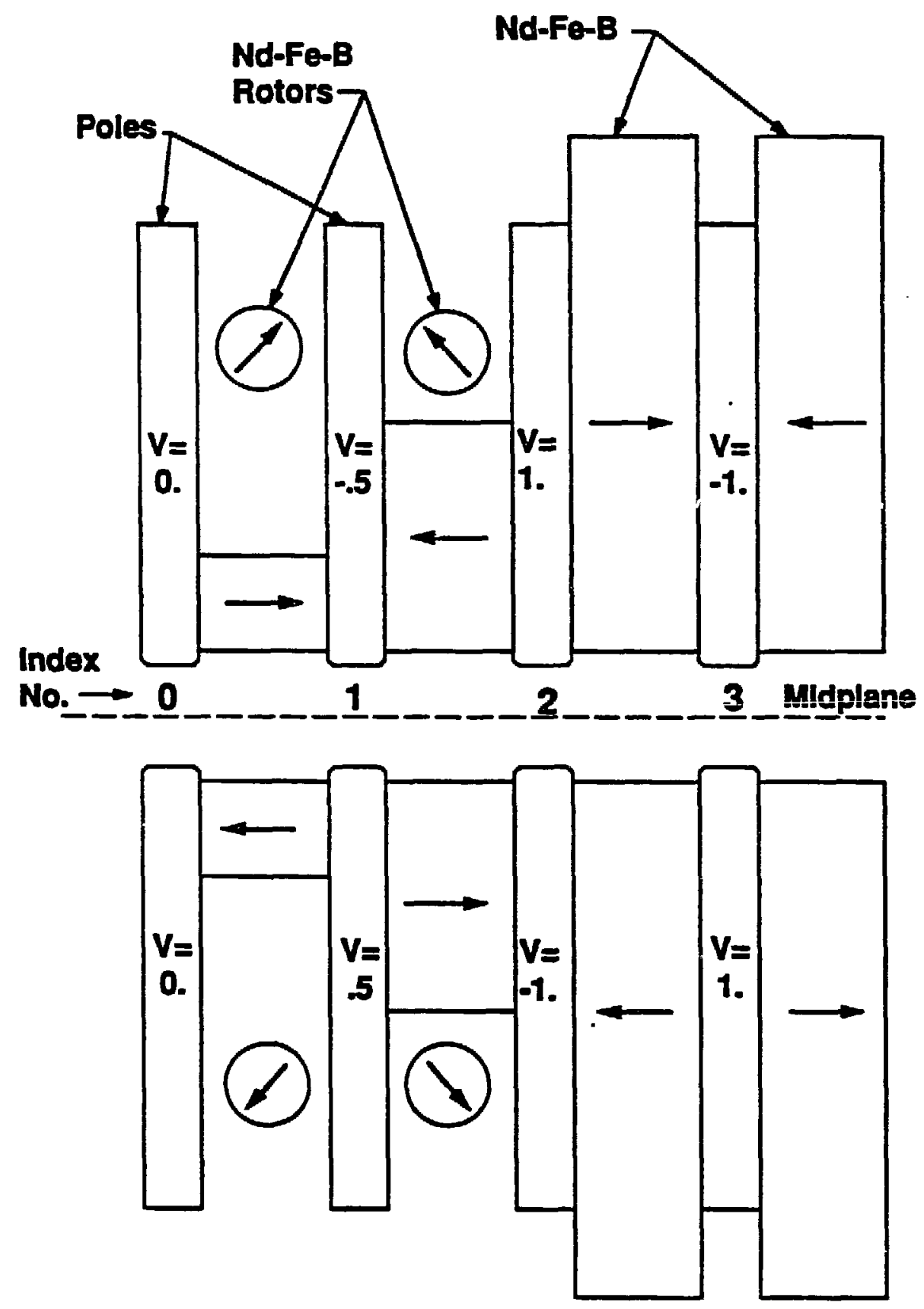

Figure 4 


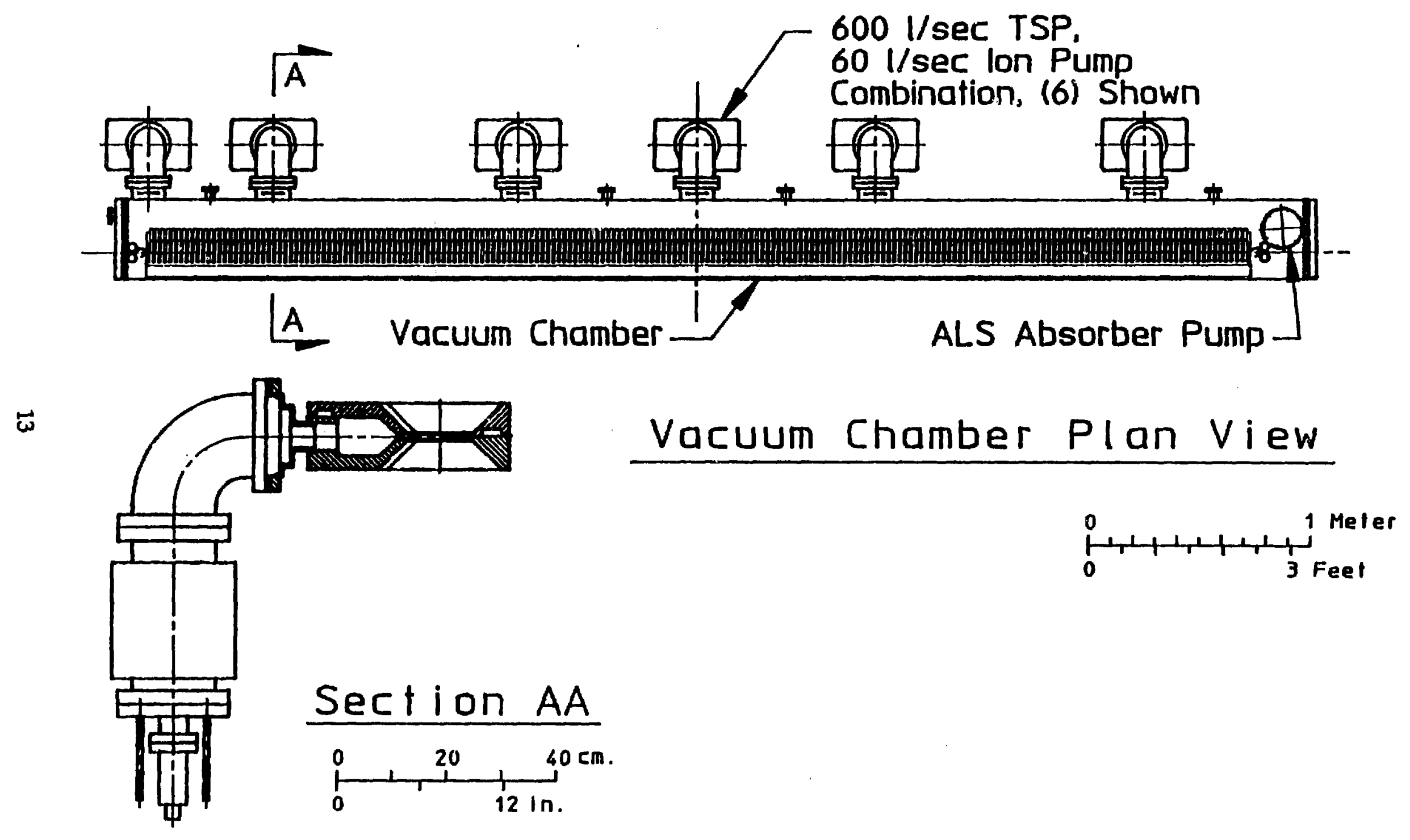

Figure 5 